Hermite polynomials on the accuracy of heartbeat representation. Tests were run fitting the heartbeats of the MIT-BIH arrhythmia database with a number of polynomials ranging from 2 to 20 . Three different strategies to determine the heartbeat's position were used. The fitting errors are reported here. Based on these results, some guidelines to choose a suitable number of Hermite polynomials for different applications are given.

\section{Didactic Speech Synthesizer: Acoustic Module Formants Model}

\author{
João Paulo Teixeira and Anildo P. Fernandes \\ Polytechnic Institute of Bragança, Bragança, Portugal
}

Keywords: Speech Analysis, Speech Synthesis, Text-To-Speech Conversion, Signal Processing, Formants Model.

Abstract: Text-to-speech synthesis is the main subject treated in this work. It will be presented the constitution of a generic text-to-speech system conversion, explained the functions of the various modules and described the development techniques using the formants model. The development of a didactic formant synthesiser under Matlab environment will also be described. This didactic synthesiser is intended for a didactic understanding of the formant model of speech production.

\section{Identification of Molecular Properties Coding Areas in Rat's Olfactory Bulb by Rank Products}

Raquel Santano-Martínez ${ }^{1}$, Raquel Leiva-González ${ }^{1}$, Milad Avazbeigi ${ }^{2,1,3}$, Agustín González-Gutiérrez ${ }^{2,1}$ and Santiago Marco 2,1

1 Universitat de Barcelona, Barcelona, Spain

2 Institute for Bioengineering of Catalonia, Barcelona, Spain

3 European Center for Soft Computing, Barcelona, Spain

Keywords: Olfaction, Odour Coding, Feature Selection, Olfactory Bulb, Chemotopy, 2-Deoxyglucose Uptake.

Abstract: Neural coding of chemical information is still under strong debate. It is clear that, in vertebrates, neural representation in the olfactory bulb is a key for understanding a putative odour code. To explore this code, in this work we have studied a public dataset of radio images of 2-Deoxyglucose uptake (2-DG) in the olfactory bulb of rats in response to diverse odorants using univariate pixel selection algorithms: rank-products and Mann-Whitney $\mathrm{U}$ (MWU) test. Initial results indicate that some chemical properties of odorants preferentially activate certain areas of the rat olfactory bulb. While non-parametric test (MWU) has difficulties to detect these regions, rank-product provides a higher power of detection.

\section{Does Low B-value can Handle Q-ball and DTI Reconstructions? \\ Diffusion MRI Experiment of Ex-vivo Pigs Spinal Cord Phantom}

\footnotetext{
Aleksandra Klimas ${ }^{1}$, Kamil Gorczewski ${ }^{2}$, Przemysław Pencak $^{3}$, Zofia Drzazga ${ }^{1}$ and Uwe Klose ${ }^{2}$

${ }^{1}$ University of Silesia, Katowice, Poland

2 University of Tuebingen, Germany

${ }^{3}$ Mielecki University Hospital of Silesian Medical University, Katowice,
} Poland

Keywords: Q-ball Reconstruction, Diffusion Tensor Imaging, Diffusion Phantom, Fibre Crossing.

Abstract: The direction of axons in white matter can be estimated using a deterministic fibre tracking algorithms and diffusion weighted imaging. The aim of this work was to evaluate the data, obtained from pig spines phantom measurements with relatively low b-value, using two types of reconstructions: diffusion tensor imaging (DTI) and q-ball approach. Pigs spines submerged in agar gel were used to prepare a phantom with two crossing populations of fibres. The phantoms were measured in $3 T$ MR scanned for b-value of 1000 and $2000 \mathrm{~s} / \mathrm{mm} 2$ for q-ball and 200-2000s/mm2 for DTI reconstruction. Analysis of crossing and single fibre population regions in the scanners showed that the median dispersions from the reference directions in case of single fibre population were c.a. $4 \hat{A}^{\circ}$ and for crossing area c.a. $12^{\circ}$ and $6.5^{\circ}$ for b-value of $1000 \mathrm{~s} / \mathrm{mm} 2$ and $2000 \mathrm{~s} / \mathrm{mm} 2$ respectively. The q-ball approach was able to resolve crossing problem for both low b-values. It was shown here that coherent results can be achieved even with lower b-values than proposed by the theory.

\begin{tabular}{|lr|}
\hline $\begin{array}{l}\text { Poster Session } 4 \\
10: 40-11: 30\end{array}$ & HEALTHINF \\
\hline
\end{tabular}

\section{Evaluation of a Web-based Mentor Functional System for Employment Support Training of Nurses who Have Not been Employed}

\author{
Yumiko Nakamura ${ }^{1}$, Yukie Majima ${ }^{2}$, Yasuko Maekawa², \\ Kaori Fukayama ${ }^{1}$ and Kazumi Hoshi ${ }^{1}$ \\ 1 Osaka Prefecture University, Habikino Osaka, Japan \\ 2 Osaka Prefecture University, Sakai Osaka, Japan
}

Keywords: Nurse, Employment Support Training Program, Mentor Functional System, e-Learning.

Abstract: We developed a mentor functional system combined with an e-learning training program to support nurses who have not been employed. Subsequently, to evaluate it, we conducted a survey using a constitutive questionnaire and a group interview of 14 monitors who had used it for a month. In the mentor functional system, the questions they asked were related exclusively to nursing techniques and new information, showing that their concern for reemployment had been reduced by taking counseling from the mentor. From these observations, it follows that the mentor functional system is effective.

\section{Assistive Technology Divide A Developing Country Perspective}

\section{Allen Nnanwuba Adum, Uche Patricia Ekwugha and Mercy Aguocha \\ Nnamdi Azikiwe University, Awka, Nigeria}

Keywords: Assistive Technology Divide, Visually Impaired, Specialized ICTs.

Abstract: Potentially, Information and Communication Technologies (ICTs) can shape our individual and collective lives. Access to information, in relation to physical challenge, especially visual impairment, is a problematic issue. This is more so, in the light of digital divide. The question now is who among visually impaired adults in developing countries are aware of; have physical access to, and the resources and skills needed to use assistive digital and information technologies? Against this backdrop, this study explored awareness, accessibility and use of specialized ICTs among visually impaired adults in selected urban cities in Southeastern Nigeria. Thirty In-depth Interviews were conducted to collect data from purposively chosen visually impaired students and government employees. The study established a general awareness of ICTs, and limited awareness of specialized ICTs, among these visually impaired persons. It also found that ownership and access to these specialized ICTs, among these persons, were almost equal to zero; while the use of specialized ICTs 